
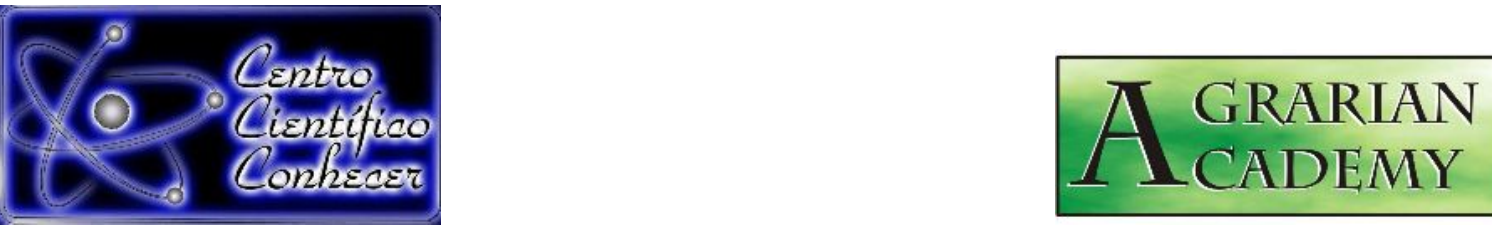

\title{
USOS, EFEITOS E POTENCIAL TÓXICO DOS AGROTÓXICOS NA QUALIDADE DO SOLO
}

\footnotetext{
José Lucas dos Santos Oliveira ${ }^{1}$, Ana Carla Bezerra de Lima ${ }^{2}$, Daniela Minini $^{3}$, Edevaldo da Silva ${ }^{4}$

${ }^{1}$ Mestrando do Programa de Pós-Graduação em Desenvolvimento e Meio Ambiente, Universidade Federal da Paraíba (lucasoliveira.ufcg@gmail.com) João Pessoa, Paraíba, Brasil.

${ }^{2}$ Mestranda do Programa de Pós-Graduação em Ciências Florestais, Universidade Federal do Espirito Santo, Jerônimo Monteiro, Espirito Santo, Brasil.

${ }^{3}$ Bolsista de Apoio Técnico de Nível superior do Programa de Pós-Graduação em Ciências

Florestais, Universidade Federal do Espirito Santo, Jerônimo Monteiro, Espirito Santo, Brasil.

${ }^{4}$ Professor da Universidade Federal de Campina Grande e do Programa de Pós-Graduação em Desenvolvimento e Meio Ambiente, Universidade Federal da Paraíba, Patos, Paraíba, Brasil.

\section{Recebido em: 14/07/2018 - Aprovado em: 28/07/2018 - Publicado em: 31/07/2018} DOI: 10.18677/Agrarian_Academy_2018a45
}

O uso de defensivos agrícolas constitui uma das principais ações que resultam na contaminação do solo e da água. A contaminação do solo ocorre após a infiltração de substâncias químicas com potencial nocivo, causando alterações na sua fertilidade e composição. Esta pesquisa teve por objetivo reportar e discutir informações compiladas da literatura científica sobre os usos, efeitos e teor tóxico dos agrotóxicos no solo. Trata-se de uma revisão bibliográfica predominantemente de publicações dos últimos 10 anos. Os estudos encontrados apontam que o uso intensivo, inadequado, com ausência de treinamento, informação e fiscalização relativos aos agrotóxicos pode contribuir e intensificar o processo de contaminação do solo. Embora a agricultura precise ser modernizada constantemente, a preocupação com o meio ambiente deve ser constante, uma vez que o uso de produtos químicos, principalmente na agricultura, contribui para a contaminação do solo e da água, comprometendo a qualidade dos alimentos que são produzidos na área contaminada. Existe uma ampla quantidade de pesquisas publicadas na literatura sobre a contaminação do solo por agrotóxicos, principalmente, aquelas relacionadas aos estudos ecotoxicológicos, em busca de conhecer como diferentes espécies reagem a um ambiente contaminado. Pesquisas que abordem temáticas voltadas à poluição dos solos por agrotóxicos são importantes para que se possa conhecer melhor quais são os impactos causados pelo seu uso em diferentes regiões e níveis de concentrações.

PALAVRAS-CHAVE: Agroquímicos; Contaminação; Problemas Ambientais. 


\title{
USES, EFFECTS AND TOXIC POTENTIAL OF AGROCHEMICALS IN QUALITY OF SOIL
}

\begin{abstract}
The use of pesticides is one of the main actions that result in contamination of soil and water. Soil contamination occurs after infiltration of harmful chemicals, causing changes in fertility and composition. This research aimed to report and discuss information compiled from the scientific literature on the uses, effects and toxic content of pesticides in soil. It is a bibliographical review predominantly of publications of the last ten years. The studies found that the intensive, inadequate use, with absence of training, information and inspection related to pesticides can contribute and intensify the process of soil contamination. Although agriculture needs to be modernized constantly, concern for the environment must be constant, since the use of chemicals, especially in agriculture, contributes to soil and water contamination, compromising the quality of food contaminated area. There is a large amount of research published in the literature on the soil contamination by pesticides, especially those related to ecotoxicological studies, in order to know how different species react to a contaminated environment. Research that addresses issues related to soil pollution by pesticides is important so that the impacts caused by their use in different regions and levels of concentrations can be better understood.
\end{abstract}

KEYWORDS: Agrochemicals; Contamination; Environmental Problems.

\section{INTRODUÇÃO}

Nas últimas décadas foi possível observar o surgimento de mudanças relacionadas a preocupação da sociedade em relação às questões ambientais, ações humanas têm causado excessiva degradação e exploração do meio ambiente (PEREIRA; GIBBON, 2014). As atividades antrópicas, incluindo o consumismo, têm acelerado a degradação dos solos, aumentando a concentração de compostos químicos no ambiente, tal como os agrotóxicos, derivados de petróleo, entre outros, comprometendo a saúde do meio e dos organismos que habitam esse ecossistema (ANDRÉA, 2010).

Agrotóxicos são produtos químicos comercializados com objetivo de contribuir com os processos de produção agrícola, principalmente, em larga escala, sendo ainda definidos pelo Ministério do Meio Ambiente, como agentes de processos químicos, físicos e biológicos (BRASIL, 2018). Os agrotóxicos atuam na prevenção e controle de pragas nas plantações, em contrapartida, surgem, com a utilização dessa prática, riscos que comprometem tanto o meio ambiente, como também, os indivíduos que tenham alguma forma de contato direto ou indiretamente com esses produtos químicos (VALENZUELA et al., 2011).

Os problemas provenientes de contaminações por agrotóxicos podem acontecer em pequenas ou grandes concentrações, por muito deles possuírem alto potencial de toxicidade ambiental, sendo necessário, antes do uso, o conhecimento adequado sobre as características químicas desses agroquímicos (ANDRÉA, 2010). As culturas agrícolas, sejam para alimentação ou para a geração de matéria prima para indústrias, apresentam altos riscos ocupacionais, relacionados à intoxicação por agrotóxicos de uma comunidade que viva na região, problemas de saúde crônicos e reprodutivos, além de impactos ecológicos diversos (FARIA et al., 2007).

Estima-se que trabalhadores de países em desenvolvimento sofrem cerca de 70 mil intoxicações anualmente, alguns casos considerados mais graves, levam, inclusive, a óbito, acompanhados de outros cerca de sete milhões de doenças não 
fatais que tem como uma de suas causas primárias, a exposição a produtos químicos usados em plantios (CASTRO et al., 2009).

O Brasil é considerado um dos líderes mundiais em cultivares agrícolas, que realizam algum tipo de uso de produtos químicos. O Brasil, em 2010, arrecadou cerca de US $\$ 7,3$ bilhões em vendas de agroquímicos para plantios, cerca de $7 \%$ de todas as vendas no setor agropecuário do país e, entre 1990 e 2010, o país cresceu $576 \%$ no setor agroquímico, enquanto que, no mesmo intervalo de tempo, o mercado mundial cresceu 83\% (SILVA, COSTA, 2012).

Essas práticas podem levar à contaminação e infertilidade do solo, contaminação dos reservatórios aquáticos, entre outros, tornando mais improdutivo ao longo do tempo e/ou aumentando o custo da manutenção, limitando assim o uso dos recursos e potencial para a produtividade agrícola (SOUZA et al., 2015).

Por isso, são necessários mais estudos sobre as vantagens e desvantagens do uso dos agroquímicos, assim como, conhecer melhor quais os riscos no uso, e a legislação vigente que determine e regulamente sua utilização, promovendo assim um consumo mais consciente dos usuários, e um controle mais eficaz na comercialização de tais produtos. Esta pesquisa teve por objetivo reportar e discutir informações compiladas da literatura científica sobre os usos, efeitos e teor tóxico dos agrotóxicos no solo.

\section{Poluição do solo}

O conceito de solo é definido por diversos profissionais (ecólogos, pedólogos, físicos, agrônomos, biólogos), sendo a camada mais superficial da Terra, com a função de sustentar diversas formas de vida, constituído por uma parte orgânica (derivada de decomposição de animais e plantas, macrofauna, mesofauna e microfauna) e outra inorgânica, tais como fragmentos de rochas (LEPSCH, 2010).

Segundo a Lei 6.938/81 da Política Nacional de Meio Ambiente, a poluição é a deterioração da qualidade ambiental resultante de atividades que direta ou indiretamente prejudiquem a saúde, segurança e bem-estar da população e afetem desfavoravelmente a biota, os recursos ambientais, a atmosfera, as águas interiores, superficiais e subterrâneas, os estuários, o mar territorial, o solo, o subsolo e os elementos que constituem a biosfera (BRASIL, 2010).

De maneira mais restrita, o Decreto oㅜ 28.687/82 (BRASIL, 1982), no art. 72 define poluição do solo e do subsolo como a deposição, disposição, infiltração, acumulação, injeção de substâncias ou produtos poluentes no estado sólido, líquido ou gasoso, que provoquem alteração nas características, e comprometa o uso e torne prejudicial ao homem e a outros organismos. Pela ação do homem, os solos podem sofrer alterações físicas resultantes de atividades agrícolas (aragem, gradagem, compactação), queimadas, erosão, impermeabilização e de caráter químico como fertilização artificial, salinização, aplicação de pesticidas, disposição de resíduos sólidos e líquidos (LEMOS, MUSAFIR, 2014).

Com o aumento populacional e a escassez de alimentos no mundo, algumas práticas como o manejo intensivo do solo, a monocultura e o uso de agrotóxicos e fertilizantes químicos tornaram-se comuns para o aumento da produção de alimentos. Os principais objetivos no uso desses agroquímicos é a disponibilização e o aumento do suprimento de nutrientes e correção do pH do solo (fertilizantes e corretivos) e a proteção das lavouras pelo controle de pragas e doenças (defensivos agrícolas) (ARAÚJO, MONTEIRO, 2007).

A utilização destas práticas pode, entretanto, causar diminuição na qualidade do solo e a degradação química, como consequência da acumulação de elementos 
químicos e compostos em níveis acima do tolerável (ARAÚJO, MONTEIRO, 2007). Os agroquímicos trouxeram benefícios inestimáveis para o cultivo de muitas culturas. Entretanto, o que se tem discutido são os malefícios advindos do uso repetitivo e excessivo, que poluem o solo com contaminantes (metais tóxicos, como o chumbo) e comprometem a qualidade do ecossistema, e por consequência a saúde humana, por não serem digeridos por organismos vivos (FREITAS et al., 2009; ANTONIOLLI et al., 2013).

\section{Agrotóxicos: Classificação e pesquisas toxicológicas}

Os agrotóxicos são substâncias químicas ou suas misturas, que apresentam finalidade, direta ou indireta, de prevenir, repelir ou acabar, com agentes infecciosos, nocivos e prejudiciais aos cultivos e criações (PERES et al., 2003; ZIMBA; ZIMUDZI, 2016).

Logo após a Segunda Guerra Mundial, houve um aumento de substâncias inseridas no uso extensivo na agricultura, sendo 0 Dicloro-DifenilTricloroetano (DDT), um dos produtos químicos mais aplicados em larga escala nas lavouras para combater insetos.

Segundo o Ministério do Meio Ambiente - MMA (BRASIL, 2018), os defensivos agrícolas podem ser classificados em duas categorias, onde ambos são liberados para uso comercial pelo Ministério da Agricultura, Pecuária e Abastecimento e da Saúde, são elas: agrícolas - utilizados para setores de produção, armazenamento e beneficiamento de produtos agrícolas, em pastos e reflorestamentos; e não agrícolas - utilizados no meio ambiente nativo e em meio urbano como, por exemplo, em tratamentos de água (são liberados pelo Ministério da Saúde).

Os agrotóxicos são divididos em diferentes classes de acordo com a finalidade, podendo ser: herbicidas, fungicidas, algicidas e inseticidas (Quadro 1). Os agrotóxicos também podem ser classificados em função da DL50 (dose letal), que é expressa em miligramas de princípio ativo por quilogramas de peso vivo ( $\mathrm{mg} \mathrm{kg}^{-1}$ ) que constitui a quantidade de cada determinada substância que possa provocar a mortalidade de $50 \%$ de espécies utilizadas em testes experimentais (WHO, 2018).

No Brasil, a classificação toxicológica dos agrotóxicos é feita de acordo com as exigências da Agência Nacional de Vigilância Sanitária (ANVISA), órgão de controle do Ministério da Saúde, se dividindo em quatro classes de perigo para a saúde humana, e é representada por diferentes cores nos rótulos dos produtos: classe I (rótulo vermelho) extremamente tóxico; classe II (rótulo amarelo) altamente tóxico; classe III (rótulo azul) medianamente tóxico; classe IV (rótulo verde) pouco tóxico (ANVISA, 2018).

QUADRO 1. Algumas substâncias químicas classificadas segundo a classe de agrotóxico.

\begin{tabular}{|l|c|c|}
\hline $\begin{array}{c}\text { Classe de } \\
\text { agrotóxico }\end{array}$ & Substâncias Químicas/Extrato & Referência \\
\hline Herbicidas & $\begin{array}{c}\text { Ácido diclorofenoxiacético; } \\
\text { Glyphosate + Diuron; Propanil; }\end{array}$ & Oliveira-Junior et al. (2011) \\
\hline Fungicidas & Triticonazol; Tiofanato metílico & $\begin{array}{c}\text { Garcia et al. (2016) } \\
\text { Rampazzo e Blum (2014) }\end{array}$ \\
\hline Algicidas & Diuron & Pedro et al. (2014) \\
\hline Inseticidas & $\begin{array}{c}\text { Extrato de Nicotiana tabacum e de } \\
\text { Azadirachta indica }\end{array}$ & Aguirre et al. (2016) \\
\hline
\end{tabular}


A manipulação de agrotóxicos precisa ser cautelosa, atendendo determinações técnicas previstas em lei (no 7.802/89) e com classificação de utilização, por apresentarem teor significativo de toxicidade (RODRIGUES, 2006). Acredita-se que controlando a população dos vetores com o uso de defensivos agrícolas, minimiza-se o risco da perda total da safra, seja ela causada por doença ou praga (ARAÚJO et al., 2015).

A agricultura precisa ser modernizada constantemente, mas com atenção ao uso adequado dos recursos naturais. O modelo adotado nos anos 60-70 apresentava facilidades concedidas pelo governo federal, como o crédito rural para aquisição de tecnologias externas, tais como: agrotóxicos e fertilizantes (BALSAN, 2006).

Atualmente, o Brasil é um dos maiores produtores de alimentos no mundo e, também, um dos países que mais consome agrotóxicos nos métodos de produção de diversas culturas agrícolas, desenvolvendo tecnologias que atendam as demandas de exportação (CARVALHO et al., 2017). No Quadro 2, são relacionados os agrotóxicos utilizados para algumas das culturas agrícolas. Em algumas situações um só tipo de agrotóxico não é suficiente para promover o controle de algumas infestações, sendo necessário mistura de diferentes tipos de agrotóxicos (GAZZIERO, 2015).

O uso intensivo de agrotóxicos pode contribuir para processos de contaminação do solo e da água (DELLA VECHIA et al., 2016). O uso inadequado, ausência de treinamento, informação, e fiscalização pode intensificar esses processos de contaminação ambiental (BUTINOF et al., 2015). Na saúde humana, os efeitos podem ser diversos, a depender das características do produto e tempo de exposição, podendo em longo prazo, causar distúrbios neurológicos ou imunológicos, como também câncer, seja por exposição direta ao agrotóxico ou consumo de alimentos contaminados com grandes quantidades desse produto (DELLA VECHIA et al., 2016).

QUADRO 2. Principais agrotóxicos usados para produção agrícola publicados na Literatura (2011-2017).

\begin{tabular}{lccc}
\hline \multicolumn{1}{c}{ Cultivar } & Agrotóxico & $\begin{array}{c}\text { Finalidade } \\
\text { de uso }\end{array}$ & Referência \\
\hline Acelga & Astro & Inseticida & Silva et al. (2011) \\
Arroz & Clomazone & Herbicida & Schreiber et al. (2015) \\
Cana-de-açúcar & Propiconazol & Fungicida & Chapola et al. (2014) \\
Algodão & Halosufuron-methyl & Herbicida & Silva et al. (2014) \\
Café & Thiodan & Inseticida & Marcon et al. (2014) \\
Algodão & Diflubenzuron & Inseticida & Barata-Silva et al. (2015) \\
Milho & Tiametoxam & Inseticida & Nörnberg et al. (2016) \\
Tomate & Metribuzina & Herbicida & Rodríguez e Plaza (2016) \\
Cana-de-açúcar & Indaziflam & Herbicida & Amim et al. (2016) \\
Feijão & Fomesafen & Herbicida & Marchioretto e Magro (2017) \\
\hline
\end{tabular}

O processo de retenção, transporte e modificação dos agrotóxicos após entrarem em contato com o ambiente, dependem intrinsecamente de fenômenos de sorção, que estão diretamente relacionados a processos de lixiviação, e, estes, podem alcançam os recursos hídricos próximos ao local de descarga, causando sua contaminação (MARCHETTI; LUCHINI, 2004).

No Semiárido brasileiro está concentrada uma parcela considerável da fruticultura do Brasil, nessa prática agrícola encontram-se altas concentrações de AGRARIAN ACADEMY, Centro Científico Conhecer - Goiânia, v.5, n.9; p.458 2018 
defensivos agrícolas, afetando principalmente o solo da região e seus processos bioquímicos, e chegam por aplicação direta ou por atividades indiretas.

Algumas regiões do Cerrado brasileiro não apresentam solo muito fértil para prática da agricultura assim, há um uso intenso de agrotóxicos nas atividades de campo nessa região, gerando mais problemas quanto a contaminações do solo e/ou reservatórios de água (SOARES; PORTO, 2007).

Santos et al. (2017), relatam que os agrotóxicos e seus compostos apresentam sérios riscos ao organismo humano podendo interferir na saúde. Já o estudo de Viero et al. (2016), aponta que os pesticidas apresentam maiores riscos aos trabalhadores rurais e ao meio onde estão sendo depositados devido ao contato direto.

Atualmente, há diversas pesquisas que reportam avaliações de efeitos tóxicos de agrotóxicos para várias culturas agrícolas (Quadro 3). Melo et al., (2010) observaram que o tipo de agrotóxico assim como as concentrações utilizadas e a quantidade de matéria orgânica em plantios influencia o solo de acordo com as características físicas e químicas, bem como a persistência residual. No estudo de Niero (2007) foi analisado Pyraclostrobin e Fluquinconazole + carbendazim, fungicidas, que reduziram as doenças fúngicas e suas intensidades em uma produção de sementes e a ocorrência da doença não afetou a produção de soja ao final do ciclo.

Por fim, Antoniolli et al. (2013) o incremento das dosagens de glifosato e epoxiconazol contribuiu para o aumento no número de insetos da Ordem Collembola, e do gênero Isotomidae, correlacionado ao aumento das dosagens dos respectivos agrotóxicos e, não apresentaram efeitos inibitórios para 0 estabelecimento desses organismos no solo.

QUADRO 3. Agrotóxicos observados em estudos na produção agrícola publicado na literatura (2003-2013).

\begin{tabular}{|c|c|c|c|}
\hline Composto & Concentração* & Efeitos observados & Referência \\
\hline $\begin{array}{l}\text { Inseticida } \\
\text { (Malathion) }\end{array}$ & $\begin{array}{c}500 \text { CE }(2 \text { e } 4) \\
\text { mg de i.a./mL }\end{array}$ & $\begin{array}{l}\text { Malathion foi medianamente seletivo } \\
\text { em favor de ninfas de } 5^{\circ} \text { instar ( } 49,38 \% \\
\text { de mortalidade) e não seletivo em favor } \\
\text { de ninfas } 30 \text { instar e adultos ( } 95,34 \text { e } \\
88,99 \% \quad \text { de mortalidade, } \\
\text { respectivamente) do Podisus rostralis. }\end{array}$ & $\begin{array}{l}\text { Gonring et } \\
\text { al. (2003) }\end{array}$ \\
\hline $\begin{array}{c}\text { Fungicida } \\
\text { (Fluquinconazol } \\
e \\
\text { +carbendazim) } \\
\\
\text { (Pyraclostrobin) }\end{array}$ & $\begin{array}{l}\text { 0,250 +62,5 } \\
\text { g i.a.ha }{ }^{-1} \\
75 \text { g i.a.ha }{ }^{-1}\end{array}$ & $\begin{array}{l}\text { Apresentaram menor severidade da } \\
\text { doença quando aplicados em } \\
\text { sementes de soja antes da plantação. }\end{array}$ & Niero (2007) \\
\hline $\begin{array}{l}\text { Herbicidas } \\
\text { (Sulfentrazone, } \\
\text { Oxyfluorfen e } \\
\text { Isoxaflutole) }\end{array}$ & $\begin{array}{l}0,5,0,72 \text { e } 0,113 \\
\mathrm{~kg} \mathrm{ha}^{-1}, \\
\text { (respectivamente) }\end{array}$ & $\begin{array}{l}\text { As características inerentes a cada } \\
\text { herbicida, bem como entre os solos, } \\
\text { influenciam em sua persistência. O } \\
\text { sulfentrazone apresentou elevado } \\
\text { efeito residual nos três solos } \\
\text { estudados. }\end{array}$ & $\begin{array}{l}\text { Melo et al. } \\
(2010)\end{array}$ \\
\hline $\begin{array}{c}\text { Fungicida } \\
\text { (Epoxiconazol) } \\
\text { Herbicida } \\
\text { (Glifosato) }\end{array}$ & $\begin{array}{c}0,75 \text { e } 1,5 \mathrm{~L} \mathrm{ha}^{-1} \\
2 \text { e } 4 \mathrm{~L} \mathrm{ha}{ }^{-1}\end{array}$ & $\begin{array}{l}\text { O número de colêmbolos apresentou } \\
\text { incremento com o aumento da } \\
\text { dosagem dos agrotóxicos. }\end{array}$ & $\begin{array}{l}\text { Antoniolli et } \\
\text { al. (2013) }\end{array}$ \\
\hline
\end{tabular}


Agrotóxicos possuem efeitos nocivos à saúde humana como por exemplo, elevado potencial carcinogênico, mutagênico, ou ainda podem influenciar, em algum nível, no desequilíbrio hormonal, e, por isso, podem ter a comercialização proibida no Brasil (BRASIL, 2002). Ingredientes ativos nos agrotóxicos que possam causar intoxicação humana ou contaminação ambiental, dentre outros impactos, também podem ser reavaliados pela Agência Nacional de Vigilância Sanitária e ter a comercialização proibida, como, por exemplo, o cihexatina, endossulfam, forato e carbofurano, que foram proibidos de serem comercializados nos anos de 2009, 2010, 2015 e 2017, respectivamente (ANVISA, 2018).

A venda de agrotóxicos no Brasil é regida pela Lei 9.802/1989 (BRASIL, 1989) e pelo Decreto 4.074/2002 que determina que, para a comercialização dos agrotóxicos, deve existir registro específico que permita a venda por proprietários e/ou empresas que fabriquem os agrotóxicos, além dos usuários terem que devolver os recipientes vazios em até um ano no local de compra para destinação final correta (BRASIL, 2002). Esse registro deve ser emitido por órgãos federais para a produção, transporte ou comercialização dos agrotóxicos, atendendo as diretrizes da ANVISA e do IBAMA, que é órgão responsável por avaliar o potencial de periculosidade ambiental dos agrotóxicos (BRASIL, 2018).

Nesse contexto, é importante o uso de técnicas agroecológicas, pois minimizam o uso de agrotóxicos sintéticos, como por exemplo, com o uso de extratos vegetais de plantas exóticas como o neem e o eucalipto, usados para controle de insetos (SANTOS; SILVA, 2015). Outro método eficaz para a erradicação de pragas em lavouras é o controle microbiológico, que consiste no uso de microrganismos como bactérias ou fungos para eliminar pragas que possam comprometer a produtividade agrícola (SOBRINHO; MOTA, 2016).

É importante destacar que a capacitação dos agricultores para o conhecimento sobre os tipos de insetos que podem estar em contato com a lavoura é necessária, isso por que é provável que existam insetos que são benéficos a lavoura e aumentam a produtividade do cultivo, mas, por falta de conhecimento dos agricultores podem vir a serem eliminados com o uso dos agrotóxicos (EMBRAPA, 2014).

Ainda nesse contexto, o Manejo Integrado de Pragas (MIP) também é importante, isso por que esse manejo contribui para a minimização de problemas que são desencadeados pelo uso de agrotóxicos convencionais, e está diretamente associado ao uso de organismos e tecnologias visando a diminuição no uso de agrotóxicos (SANTOS; SILVA, 2015). Além disso, o MIP é uma técnica que reduz o uso de agrotóxicos adotando métodos de controle de pragas alternativos que sejam eficazes para manter a produtividade agrícola, mas sem causar impactos ao ambiente que são oriundos do uso de agrotóxicos no modelo convencional de produção agrícola (EMBRAPA, 2014).

\section{CONSIDERAÇÕES FINAIS}

Diversas pesquisas publicadas na literatura abordam a problemática do uso de agrotóxicos relacionando o seu potencial tóxico com a saúde humana, como também, analisam a influência de concentrações experimentais no desenvolvimento de diferentes espécies que podem comprometer a produção agrícola para uma determinada região.

Sendo assim, as fiscalizações e o controle na comercialização desses produtos químicos são importantes, tendo em vista o seu potencial nocivo, principalmente, a saúde humana e ambiental. 
Diante desse cenário, as leis brasileiras que regulam a produção e comercialização de agrotóxicos no Brasil, bem como a fiscalização realizada por órgãos federais, são fundamentais para controlar o uso desses defensivos agrícolas nas culturas agrícolas.

Práticas agroecológicas e o Manejo Integrado de Pragas são importantes alternativas para práticas agrícolas com menos uso de agroquímicos sintéticos e para a obtenção de alimentos mais saudáveis e manutenção da qualidade ambiental.

\section{AGRADECIMENTOS}

A Coordenação de Aperfeiçoamento de Pessoal de Nível Superior (CAPES), pelo apoio ao desenvolvimento de trabalhos científicos como também pela importância que exerce prestando incentivos a produtividade acadêmica e a capacitação.

\section{REFERÊNCIAS}

AGUIRRE, C. E. D.; JUINIOR, L. M.; A.; FRAGOSO, D. F. M.; ATAÍDE, J.; O.; ACEVEDO, A. C. T.; et al. Atividade insetida de Nicotiana tabacum e Azadirachta indica sobre Helicoverpa armigera Hubner, 1908 (Lepidoptera: Noctuidae). Revista Univap, v. 22. n. 40. 2016. Disponível em: <http://revista.univap.br/index.php/revistaunivap/article/view/1042>. doi: http://dx.doi.org/10.18066/revistaunivap.v22i40.1042.

AMIM, R. T.; FREITAS, S. P.; FREITAS, I. L. J.; SCARSO, M. F. Banco de sementes do solo após aplicação de herbicidas pré-emergentes durante quatro safras de canade-açúcar. Pesquisa Agropecuária Brasileira, v. 51, n. 10, p. 1710-1719, 2016. Disponível em: <http://www.scielo.br/pdf/pab/v51n10/1678-3921-pab-51-1001710.pdf>. doi: 10.1590/S0100-204X2016001000002.

ANDRÉA, M. M. O uso de minhocas como bioindicadores de contaminação de solos. Acta Zoológica Mexicana, n. 2, p. 95-107, 2010. Disponível em: <http://www.redalyc.org/pdf/575/57515556007.pdf>. http://www.redalyc.org/articulo.oa?id=57515556007.

doi:

ANTONIOLLI, Z. I.; REDIN, M.; SOUZA, E. L.; POCOJESK, E. Metais pesados, agrotóxicos e combustíveis: efeito na população de colêmbolos no solo. Ciência Rural, v. 43, n. 6, $2013 . \quad$ Disponível em: <http://www.scielo.br/pdf/cr/2013nahead/a15813cr4635.pdf>.

ANVISA, Agência Nacional de Vigilância Sanitária (2018). Regularização de Produtos - Agrotóxicos. Disponível em: <http://portal.anvisa.gov.br/registros-eautorizacoes/agrotoxicos/produtos/reavaliacao-de-agrotoxicos>. Acesso em 28 de Jun de 2018.

ARAÚJO, A. S. F.; MONTEIRO, R. T. R. Indicadores biológicos de qualidade do solo. Bioscience Journal, v. 23, n. 3, p. 66-75, 2007. Disponível em: $<$ http://www.seer.ufu.br/index.php/\%20biosciencejournal/article/\%20viewFile/\%20668 4/4403>. 
ARAÚJO, W. L.; OliveIRA, A. G.; FERREIRA, A. P. N.; SOUSA, F. S.; ANDRADE, A. B. A. Manejo de pragas no controle de doenças no cultivo de hortícolas. Revista Verde de Agroecologia e Desenvolvimento Sustentável. Pombal, v. 10, n. 5, p. 4350, 2015.2 Disponível em: <http://gvaa.com.br/revista/index.php/RVADS/article/view/3887/3520>. doi: http://dx.doi.org/10.18378/rvads.v10i5.3887.

BALSAN, R. Impactos decorrentes da modernização da agricultura brasileira. Revista de geografia agrária, v. 1, n. 2, p. 123-151, 2006. Disponível em: <http://www.seer.ufu.br/index.php/campoterritorio/article/view/11787/8293>.

BARATA-SILVA, C.; SANTOS, T. P.; GOUVÊA, A. V.; LARENTIS, A. L.; MOREIRA, J. C.; et al. Adaptação da metodologia de análise de metemoglobina como biomarcador de efeito da exposição ao agrotóxico diflubenzuron. Química Nova, v. 38, n. 4, p. 533-537, 2015. Disponível em: <https://www.arca.fiocruz.br/handle/icict/11053>. doi: http://dx.doi.org/10.5935/01004042.20150038.

BRASIL, Ministério do Meio Ambiente. Agrotóxicos, 2018. Disponível em: <http://www.mma.gov.br/seguranca-quimica/agrotoxicos>. Acesso em: 28 de Jun de 2018.

BRASIL. Decreto número 28.687, de 11 de fevereiro de 1982. Diário Oficial da República Federativa do Brasil, Brasília, DF, n.18, 1982.

BRASIL. Decreto número 4074, de 4 de Janeiro de 2002. Disponível em: <http://www.planalto.gov.br/ccivil_03/decreto/2002/D4074.htm>. Acesso em: 28 de Jun de 2018.

BRASIL. Lei 6.938/81 - Lei da Política Nacional do Meio Ambiente, 2010. Disponível em: <http://www.planalto.gov.br/ccivil_03/LEIS/L8028.htm>. Acesso em: 28 de Jun de 2018.

BRASIL. Lei 7.802, de 11 de Julho de 1989. Disponível em: <http://www.planalto.gov.br/ccivil_03/leis/L7802.htm>. Acesso em: 28 de Jun de 2018.

BUTINOF, M.; FERNANDEZ, R. A.; STIMOLO, M. I.; LANTIERI, M. J.; BLANCO, M.; et al. Pesticide exposure and health conditions of terrestrial pesticide applicators in Córdoba Province, Argentina. Cadernos de Saúde Pública, v. 31, n. 3, p. 633-646, 2015. Disponível em: <http://www.scielo.br/pdf/csp/v31n3/0102-311X-csp-31-0300633.pdf>. doi: http://dx.doi.org/10.1590/0102-311x00218313.

CARVALHO, M. M. X.; NODARI, E. S.; NODARI, R. O. "Defensives" or "pesticides"? $A$ history of the use and perception of pesticides in the state of Santa Catarina, Brazil, 1950-2002. História, Ciências, Saúde - Manguinhos, v. 24, n. 1, p. 75-91, 2017. Disponível em: <http://www.scielo.br/scielo.php?pid=S010459702017000100075\&script=sci_arttext>. doi: http://dx.doi.org/10.1590/s010459702017000100002. 
CASTRO, V. L. S. S. Uso de Misturas de Agrotóxicos na Agricultura e Suas Implicações Toxicológicas na Saúde. Journal of the Brazilian Society of Ecotoxicology. v. 4, n. 1-3, p. 87-94, 2009. Disponível em: <file:///C:/Users/Dell/Downloads/12_jbse_v14n1Castro.pdf>. doi: 10.5132/jbse.2009.01.012.

CHAPOLA, R. G.; OGASAWARA, G. A.; JANS, B.; JUNIOR, N. S. M. Controle da podridão abacaxi da cana-de-açúcar por meio da pulverização de fungicidas em rebolos no sulco de plantio. Ciência Rural, v. 44, n. 2, p. 197-202, 2014. Disponível em: $\quad<\mathrm{http}: / /$ www.scielo.br/scielo.php?script=sci_arttext\&pid=S010384782014000200001 >. doi: http://dx.doi.org/10.1590/S0103-84782014000200001.

DELLA VECHIA, J. F.; CRUZ, C.; SILVA, A. F.; CERVEIRAJR, W. R.; GARLICH, N. Macrophyte bioassay applications for monitoring pesticides in the aquatic environment. Planta Daninha, v. 34, n. 3, p. 587-603, 2016. Disponível em: $<$ http://www.scielo.br/scielo.php?script=sci_arttext\&pid=S0100-

83582016000300597>. doi: http://dx.doi.org/10.1590/s0100-83582016340300021.

EMBRAPA, Empresa Brasileira de Pesquisa Agropecuária. Ministério da Agricultura, Pecuária e Abastecimento. 2014. Disponível em: $<$ https://www.embrapa.br/busca-de-noticias/-/noticia/1671932/embrapa-mostra-aimportancia-do-manejo-integrado-de-pragas-na-agrishow $>$. Acesso em: 30 de Jun de 2018.

FARIA, N. M. X.; FASSA, A. G.; FACCHINI, L. A. Intoxicação por agrotóxicos no Brasil: os sistemas oficiais de informação e desafios para realização de estudos epidemiológicos. Ciência \& Saúde Coletiva, v. 12, n. 1, p. 25-38, 2007. Disponível em: $\quad<\mathrm{http}: / / w w w . s c i e l o . b r / p d f / \% 0 D / c s c / v 12 n 1 / 04 . p d f>$. doi: http://dx.doi.org/10.1590/S1413-81232007000100008.

FREITAS, E. V. S.; NASCIMENTO, C. W. A.; GOULART, D. F.; SILVA, J. P. S. Disponibilidade de cádmio e chumbo para milho em solo adubado com fertilizantes fosfatados. Revista Brasileira de Ciência do Solo, v. 33, n. 6, p. 1899-1907, 2009. Disponível em: <http://www.scielo.br/pdf/rbcs/v33n6/a39v33n6.pdf>. doi: http://dx.doi.org/10.1590/S0100-06832009000600039.

GARCIA, L. C.; SAVIOLI, G. I.; GREGÓRIO, L. G.; FERREIRA, L. J.; MENDES, M. T.; et al. Características físico-químicas e tóxicas do Triticonazol. Revista Intertox de Toxicologia, Risco Ambiental e Sociedade. v. 9, n. 3, p. 36-49, 2016. Disponível em: http://www.revistarevinter.com.br/autores/index.php/toxicologia/article/view/254>.

GAZZIERO, D. L. P. Misturas de agrotóxicos em tanque nas propriedades agrícolas do Brasil. Planta Daninha, v. 33, n. 1, p. 83-92, 2015. Disponível em: <http://www.scielo.br/pdf/pd/v33n1/0100-8358-pd-33-01-00083.pdf>. doi: http://dx.doi.org/10.1590/S0100-83582015000100010. 
GONRING A. H. R.; PICANÇO, M. C.; LEITE, G. L. D.; SUINAGA, F. A.; ZANUNCIO, J. C. Seletividade de inseticidas a Podisus rostralis (stal) (heteroptera: pentatomidae) predador de lagartas desfolhadoras de eucalipto. Revista Árvore, v. 27, n. 2, p. 263268, 2003.

Disponível

em: <http://www.scielo.br/scielo.php?script=sci_arttext\&pid=S010067622003000200015> . doi: http://dx.doi.org/10.1590/S0100-67622003000200015.

LEMOS, H. M.; MUSAFIR, R. E. Poluição do solo. 2014. Disponível em: $<$ http://www.mecanica-

ufrj.educacao.ws/util/b2evolution/media/blogs/ricardo/Apost_Pol_Solos_HML_REM2014.pdf>.

LEPSCH, I. F. Formação e Conservação dos Solos. 2. ed. São Paulo: Oficina de Textos. 2010. 216 p.

MARCHETTI, M.; LUCHINI, L. C. Sorção/dessorção e mineralização do inseticida acefato em solo. Pesticidas: Revista de Ecotoxicologia e Meio Ambiente, v. 14, p. 61-72, 2004. Disponível em: < https://revistas.ufpr.br/pesticidas/article/view/3124/2497>.

MARCHIORETTO, L. R.; MAGRO, T. D. Weed control and crop selectivity of postemergence herbicides in common beans. Ciência Rural, v. 47, n. 3, p. 1-6, 2017. Disponível em: <http://www.scielo.br/scielo.php?script=sci_arttext\&pid=S010384782017000300407>. doi: Http://dx.doi.org/10.1590/0103-8478cr20160295.

MARCON, L.; FILHO, P. B. S.; LOPES, D. S.; MOUNTEER, A. H.; BENJAMIN, L. A. O inseticida Thiodan $\AA$ sobre o desenvolvimento folicular de lambaris. Ciência Rural, v. $44, \quad$ n. $12, \quad$ p. 2245-2251, 2014. Disponível em: <http://www.scielo.br/pdf/cr/v44n12/0103-8478-cr-44-12-02245.pdf>. http://dx.doi.org/10.1590/0103-8478cr20140400.

MELO, C. A. D.; MEDEIROS, W. N.; TUFFI SANTOS, L. D.; FERREIRA, F. A.; FERREIRA, G. L.; et al. Efeito residual de sulfentrazone, isoxaflutole e oxyfluorfen em três solos. Planta Daninha, v. 28, n. 4, p. 835-842, 2010. Disponível em: <http://www.scielo.br/scielo.php?pid=S010083582010000400017\&script=sci_arttext\& tlng=es>. doi: http://dx.doi.org/10.1590/S0100-83582010000400017.

NIERO, R. Eficácia dos fungicidas no controle de doenças de final de ciclo (DFC) da soja. Revista Acadêmica Ciência Animal. v. 5, n. 1, p. 19-25, 2007. Disponível em: <https://periodicos.pucpr.br/index.php/cienciaanimal/article/view/9564/9177>.

NÖRNBERG, S. D.; GRÜTZMACHER, A. D.; NAVA, D. E.; VALGAS, R. A.; OZELAME, A. L. Residual effects of pesticides in peach orchards on the maize weevil Sitophilus zeamais (Coleoptera: Curculionidae). Revista Brasileira de Fruticultura, v. $38, \quad$ n. 3 , p. 1-8, 2016. Disponível em: <http://www.scielo.br/scielo.php?script=sci_arttext\&pid=S010029452016000300402> . doi: http://dx.doi.org/10/.1590/0100-29452016017. 
OLIVEIRA-JUNIOR, R. R.; CONSTANTIN J.; INOUE, M. H. Biologia e Manejo de Plantas Daninhas. Curitiba: Omnipax, 2011. p. 348. Disponível em: <http://omnipax.com.br/livros/2011/BMPD/BMPD-livro.pdf>.

PEDRO, R.; DUTRA, R. C. L.; MORAES, J. J.; DINIZ, M. F.; MATTOS, E. C. Análise por FT-IR (UATR e PAS) de Microbicidas em Filmes Poliméricos de Tintas Comerciais. Polímeros Ciência e Tecnologia. v. 24, n. 2, p. 214-221, 2014. Disponível em: <http://revistapolimeros.org.br/doi/10.4322/polimeros.2014.041>. doi: http://dx.doi.org/10.4322/polimeros.2014.041.

PEREIRA, V. A.; GIBBON C. A. A Educação Ambiental No Ensino: Investigando As Abordagens, Percepções e Desafios na Realidade de uma Escola Pública em Rio Grande (RS). Revista Brasileira de Educação Ambiental. São Paulo. v. 9, n. 2, p. 376-394, $2014 . \quad$ Disponível em: <http://www.sbecotur.org.br/revbea/index.php/revbea/article/view/2691/2928>.

PERES, F.; MOREIRA, J. C.; DUBOIS, G. S. Agrotóxicos, saúde e ambiente: uma introdução ao tema. [IN] PERES, F.; MOREIRA, J. C. (Orgs.). É veneno ou é remédio?: agrotóxicos, saúde e ambiente. Rio de Janeiro: FIOCRUZ, 2003. p. 2141. Disponível em: <http://static.scielo.org/scielobooks/sg3mt/pdf/peres9788575413173.pdf>.

PIGNATI, W. A.; LIMA, F. A. N. S.; LARA, S. S.; CORREA, M. L. M.; BARBOSA, J. R.; et al. Distribuição espacial do uso de agrotóxicos no Brasil: uma ferramenta para a Vigilância em Saúde. Ciência \& Saúde Coletiva, v. 22, n. 10, p. 3281-3293, 2017. Disponível em: < http://www.scielo.br/pdf/csc/v22n10/1413-8123-csc-22-103281.pdf>. doi: 10.1590/1413-812320172210.17742017.

RAMPAZZO, L. S.; BLUM, L. E. B. Reação de cultivares de soja à mancha parda sob diferentes técnicas de manejo. Revista Caatinga, v. 27, n. 3, p. 115-123, 2014. Disponível em: <http://www.redalyc.org/html/2371/237132104013/>.

RODRIGUES, N. R. Agrotóxicos: Análises de Resíduos e Monitoramento. Revista Multiciência. Campinas, n. 7, 2006. Disponível em: $<$ http://www.multiciencia.unicamp.br/intro_07.htm>.

RODRíGUEZ, J. A.; PLAZA, G. Efecto de ácidos hidroxicarboxílicos en la biorregulación del estrés causado por herbicidas en el cultivo de tomate. Revista Colombiana de Ciências Hortícolas, v. 10, n. 1, p. 66-79, 2016. Disponível em: $<$ http://revistas.uptc.edu.co/index.php/ciencias_horticolas/article/view/4276>. doi: http://dx.doi.org/10.17584/rcch.2016v10i1.4276.

SANTOS, C. A. B.; SILVA, A. P. M. Viabilidade do uso de inseticidas botânicos extraídos de plantas exóticas. Educação Ambiental em Ação, n. 54, p. 2241, 2015. Disponível em: <http://www.revistaea.org/artigo.php?idartigo=2241>. Acesso em: 28 de Jun de 2018.

SANTOS, T. M. M.; SILVA, I. S.; GUILHERME, M. F. S.; COSTA, V. F.; OLIVEIRA, H. M.; et al. Pesquisas sobre agrotóxicos no contexto da Educação Ambiental. Educação Ambiental em Ação, n. 61, p. 2877, 2017. Disponível em: $<$ http://www.revistaea.org/artigo.php?idartigo=2877>. 
SCHREIBER, F.; AVILA, L. A.; SCHERNER, A.; GEHRKE, V. R.; AGOSTINETTO, D. Volatility of different formulations of clomazone herbicide. Planta Daninha, v. 33, n. 2, p. 315-321, 2015. Disponível em: <http://www.scielo.br/pdf/pd/v33n2/0100-8358pd-33-02-00315.pdf>. doi: 10.1590/0100-835820155000200017.

SILVA, L. M. F.; ARAÚJO, G. T.; SILVA, A. D. V.; JUNIOR, M. V.; CARVALHO, C. M. Levantamento dos agrotóxicos utilizados na horticultura no município de Ubajara-CE. Revista Brasileira de Agricultura Irrigada, v. 5, n. 4, p. 280-285, $2011 . \quad$ Disponível em: <http://www.inovagri.org.br/revista/index.php/rbai/article/view/92/pdf_81>. doi: 10.7127/RBAI.V5N400063.

SILVA, M. F. O.; COSTA, L. M. A indústria de defensivos agrícolas. Biblioteca Digital. BNDES Setorial v. 35, p. 233-276, 2012. Disponível em: <https://web.bndes.gov.br/bib/jspui/bitstream/1408/1513/1/A\%20set.35_A\%20ind\%C 3\%BAstria\%20de\%20defensivos\%20agr\%C3\%ADcolas_P.pdf $>$.

SILVA, R. L. M.; COSTA, A. G. F.; SOFIATTI, V. Seletividade do halosulfuron-methyl aplicado na dessecação antes da semeadura da cultura do algodoeiro. Revista Brasileira de Engenharia Agrícola e Ambiental, v. 18, n. 6, p. 640-645, 2014. Disponível em:

https://ainfo.cnptia.embrapa.br/digital/bitstream/item/157349/1/Seletividade-dohalosulfuron-methyl.pdf>. doi: 10.1590/S1415-43662014000600011.

SOARES, W. L.; PORTO, M. F. Atividade agrícola e externalidade ambiental: uma análise a partir do uso de agrotóxicos no cerrado brasileiro. Ciência \& Saúde Coletiva, v. 12, n. 1, p. 131-143, 2007. Disponível em: <http://www.scielo.br/pdf/csc/v12n1/12.pdf>. doi: http://dx.doi.org/10.1590/S141381232007000100016.

SOBRINHO, R. S.; MOTA, E. F. Diagnóstico do conhecimento de agricultores de Palmeira em Beberibe-CE sobre o controle microbiológico. Educação Ambiental em Ação, n. 55, p. 2276, 2016. Disponível em: < http://www.revistaea.org/pf.php?idartigo=2276>.

SOUZA, Â. R. L.; MACHADO, J. A. D.; DALCIN, D. Análise de estudos internacionais sobre os fatores que influenciam a decisão dos agricultores pela produção orgânica. Revista em Agronegócio e Meio Ambiente. Maringá, v. 8, n. 3, p. 563-583, 2015. Disponível em: <http://periodicos.unicesumar.edu.br/index.php/rama/article/view/3569/2653>. doi: http://dx.doi.org/10.17765/2176-9168.2015v8n3p563-583.

VALENZUELA, P. M.; MATUS, M. S.; ARAYA, G. I.; PARIS, E. Pediatria ambiental: um tema emergente. Jornal de Pediatria, v. 87, n. 2, p. 89-99, 2011. Disponível em: http://www.scielo.br/scielo.php?script=sci_arttext\&pid=S0021-75572011000200003. doi: http://dx.doi.org/10.1590/S0021-75572011000200003.

VIERO, C. M.; CAMPONOGARA, S.; CEZAR-VAZ, M. R.; COSTA, V. Z.; BECK, C. L. C. Sociedade de risco: o uso dos agrotóxicos e implicações na saúde do 
trabalhador rural. Escola Anna Nery, v. 20, n. 1, 99-105, 2016. Disponível em: <http://www.scielo.br/pdf/ean/v20n1/1414-8145-ean-20-01-0099.pdf>. doi: 10.5935/1414-8145.20160014.

WORLD HEALTH ORGANIZATION. The WHO recommended classification of pesticides by hazard and guidelines to classification 1990-1991. International Programme on Chemical Safety - IPCS. Document WHO/PCS/90.1 Rev1. Disponível em: <http://whqlibdoc.who.int/hq/1990/WHO_PCS_90.1_REV.1.pdf>. Acesso em: 16 de Jul de 2018.

ZIMBA, M.; ZIMUDZI, C. Pesticide management practices among rural market gardening farmers near Harare, Zimbabwe. South African Journal of Science, v. 112, n. 9, p. 1-5, 2016. Disponível em: <http://www.sajs.co.za/pesticidemanagement-practices-among-rural-market-gardening-farmers-near-hararezimbabwe/moses-zimba-clemence-zimudzi>.

doi:

http://dx.doi.org/10.17159/sajs.2016/20150443. 